\title{
Can Uncertainty Alleviate the Commons Problem?
}

\author{
Yann Bramoullé* and Nicolas Treich ${ }^{+}$
}

November 2007

\begin{abstract}
Global commons problems, such as climate change, are often affected by severe uncertainty. The paper examines the effect of uncertainty on pollution emissions and welfare in a strategic context. We find that emissions are always lower under uncertainty than under certainty, reflecting risk-reducing considerations. We show that uncertainty can have a net positive impact on the welfare of risk-averse polluters. We extend the analysis to increases in risk, increases in risk-aversion, and to risk heterogeneity.
\end{abstract}

JEL codes: D81, C72, Q54, H23

Keywords: Externality, Uncertainty, Climate Change, Non-Cooperative Game.

*GREEN, CIRPEE, Department of Economics, Laval University, Canada.

+ Toulouse School of Economics (LERNA, INRA), France.

The first version of the paper was written in 2004 when Yann Bramoullé was visiting the Toulouse School of Economics. The last version has benefitted from the insightful remarks of two anonymous referees. We thank Christian Gollier, Patrick Gonzales and Martin Weitzman for useful discussions together with seminar participants at the Universities of Lille, Goteborg, Padova, Toulouse, Cired and Thema (Paris), HEC (Montréal), EAERE (Budapest), Cornell, AREC (Maryland), NBER and the Harvard Kennedy School. 


\section{Introduction}

Uncertainty is generally viewed as a "bad". For instance, severe uncertainty affecting future environmental damages often raises the possibility of an environmental catastrophe. In this paper, we show that uncertainty may have a positive impact in a context of global pollution. Uncertainty can lower the incentives to pollute and make risk-averse polluters better-off.

This result may have implications for the climate change debate. The recent (and disputed) Stern review states that "climate change will reduce welfare by an amount equivalent to a reduction in consumption per head of between 5 and 20\%" (Stern et al., 2007, p. 10, Executive Summary). This represents a considerable negative impact of the climatic damage. And it also represents a considerable uncertainty about this impact. ${ }^{1}$ Moreover, there still do not exist binding international cooperation mechanisms to reduce emissions of greenhouse gases, as the Kyoto protocol has failed to involve some of the biggest pollution emitters including the United States. ${ }^{2}$ Therefore, incentives to free-ride on emissions reduction remain for some (if not most) countries. In such a strategic global pollution context, our paper suggests that the large persistent uncertainty about future climate damage impacts need not be detrimental for overall welfare in our economies.

This result has an intuitive explanation. As in many other settings, economic agents tend to lower their risk-averting efforts when uncertainty is reduced. ${ }^{3}$ In our model, the variance of damage increases with pollution. Polluters can reduce the risk faced by decreasing their own emissions. While this action is taken from a purely individual point of view, it has positive social consequences. It tends to alleviate the negative externality. Welfare may even be higher under uncertainty than under certainty when the positive effect of reduced emissions is larger than the negative effect of uncertainty. Similar effects have been documented in other contexts, but they appear to have been overlooked in studies of global pollution. ${ }^{4}$

\footnotetext{
${ }^{1}$ In his critical review, Weitzman (2007) spells out different arguments that reinforce the importance of uncertainty in the discussion on climate change. Climate science indicates that large scientific uncertainties about global warming impacts persist. For example, current estimates predict a $90 \%$ chance of global warming at the end of the century lying between $1.1^{\circ}$ and $6.4^{\circ}$ (IPCC, 2007, p. 13, Summary for Policymakers).

${ }^{2}$ Also, other big emitters like China or India have ratified the protocol but are not required to reduce emissions.

${ }^{3}$ Such substitution effects engender moral hazard in insurance situation, for instance when holders of fire insurance take less preventive actions to reduce the risk of fire; see Arrow (1963) for an early reference.

${ }^{4}$ For instance, Peltzman (1975) argues that mandating safety belts in cars makes people drive more recklessly and the number of fatal accidents may rise as a result. Similarly, Viscusi (1984) emphasizes a "lulling effect" in which child-resistant bottlecaps did not have the expected positive effects in reducing child poisenings possibly due
} 
These two features - strategic interactions and uncertainty - play a central role in most global commons situations, such as climate change. ${ }^{5}$ Each feature has been extensively studied by economists, but they are usually considered separately. ${ }^{6}$ In this paper, we look at their combined effect. We study a simple model where agents' actions impose a negative externality on others and the damage from this externality is subject to uncertainty. ${ }^{7}$ We ask whether uncertainty alleviates or aggravates the negative externality.

We develop our analysis in four parts. We first set up a symmetric game of global pollution under uncertainty, and establish some basic properties of the equilibria. A unique symmetric equilibrium often exists, but asymmetric equilibria may also appear. Second, we look at the effect of uncertainty and risk-aversion on emissions and welfare. We find that emissions are always lower under uncertainty than under certainty. We characterize for small risks when this leads to an increase in welfare. This result and further numerical simulations show that, indeed, welfare is often higher under uncertainty. We then study the effects of an increase in risk and an increase in risk-aversion. Previous insights extend with some qualifications. Third, we look at risk heterogeneity. Being the only agent to face uncertainty is a large disadvantage in equilibrium. This outcome is reversed under cooperation. And fourth, we study alternative formulations of our game. We find that our main results hold under different assumptions, including uncertain benefits and two periods.

Few other papers have examined the effect of uncertainty in a strategic context. Gradstein et al. (1992) argue that the comparative statics of uncertainty are generally ambiguous. Sandler and Sterbenz (1990) look at the exploitation of a stock resource, and show that uncertainty on the size of the stock leads risk-averse firms to reduce their exploitation effort compared to certainty; see also Sandler et al. (1987). More recently, Eso and White (2004) introduces uncertainty in auctions and White (2004) introduces uncertainty in a Rubinstein bargaining model. Both

to a reduction in parental caution. See also Viscusi (1994).

${ }^{5}$ Other prominent examples include the prevention of nuclear proliferation, the prevention and containment of new diseases outbreaks, management of fish stocks, and the production of scientific knowledge, see Barrett (2007).

${ }^{6}$ Experts identified five main qualitative features to the problem of climate change, see IPCC (1995). Strategic interactions and uncertainty constitute two of these features. The remaining three are the asymmetric distribution of impacts, the long time horizon, and potential irreversibilities. We briefly discuss these issues in section V.

${ }^{7}$ Our paper is related to two branches of the literature on negative externalities. First, some papers have examined (usually numerically) the effect of learning on non-cooperative emissions (Hammitt and Adams, 1996; Ulph and Ulph, 1996; Ulph and Maddison, 1997; Baker, 2005). Second, other papers have studied the effect of uncertainty and learning on international environmental agreements (Na and Shin, 1998; Ulph, 2004; Kolstad, 2005; Boucher and Bramoullé, 2007). 
papers study the effect of uncertainty on decisions and welfare and show that uncertainty may be beneficial for risk-averse players. Our paper complements these studies. We provide the first analysis of the effect of uncertainty and risk-aversion on global pollution. ${ }^{8}$

Our model relies on two main assumptions. We discuss their plausibility in the context of our lead example, climate change. First, we assume that the variance of damage is increasing in overall pollution. This assumption is very likely satisfied in the case of climate change. For instance, Table 1 presents current scientific predictions on the effects of the atmospheric level of $\mathrm{CO} 2$ on global mean temperature at equilibrium. Clearly as the level of $\mathrm{CO} 2$ rises, the range of predicted values expands. In turn, economic damage and its variance are expected to increase with mean temperature. ${ }^{9}$ Thus, more CO2 emissions likely lead to greater and riskier damages.

\section{Insert Table 1 about here}

Our second main assumptions is that polluters, such as countries in a climate change setting, are risk-averse. This may seem more controversial. Economists often view countries as riskneutral, due to their sizes and to the possibility to pool independent risks across the population (Arrow and Lind, 1970). However, the unique features of climate change challenge this view. Risksharing opportunities are limited due to the high correlation of climate risks within communities. And potential damages are large, involving a sizable portion of the economies even in conservative estimates. Thus, Heal and Kriström (2002) recommend to better account for uncertainty and risk-aversion in the economic approach to climate change. ${ }^{10}$ In the same vein, economists working on integrated assessment models usually assume risk-aversion, see e.g. Nordhaus (1994) and Stern et al. (2007). However, these models generally neglect the externality dimension, which plays a central role here.

\footnotetext{
${ }^{8}$ Eso and White (2004) consider an additive risk which is contingent on the outcome of the game. It is only faced by the agent who wins the auction. White (2004) considers both additive and mutliplicative risks in a two-player setting. In contrast, in our game, the risk is multiplicative and faced by all agents. We study additive risks in section $\mathrm{V}$.

${ }^{9}$ See e.g. Figure 6.6 (p. 195) and Table 13.2 (p. 295) in Stern et al. (2007). See also Figure 1 in Azar and Rodhe (1997).

10 "The point is that uncertainty, risk and our attitudes towards risk really do matter in making policy decisions. They should be taken explicitly into account in formulating policy on climate change. Our final policy analysis may be as sensitive to attitudes towards risk as to some aspects of the scientific data which we work so hard to generate. Yet we have done little to introduce these issues into the policy debate." Heal and Kriström (2002, p. $16)$.
} 
The remainder of the paper is organized as follows. In section II, we introduce the model of global pollution under uncertainty, and examine its basic properties. In section III, we look at the effect of uncertainty and risk-aversion on emissions and welfare. In section IV, we study risk heterogeneity. We consider extensions in section V, and conclude in section VI.

\section{Model and Equilibrium Properties}

In this section, we introduce our model of global pollution under uncertainty, and derive some properties of the equilibria. Consider the following $n$-player game with $n \geq 2$. Each agent (or country) $i$ emits a level of pollution $e_{i} \geq 0$. Agents benefit from emitting pollution, but suffer from the global level of pollution $\sum_{j=1}^{n} e_{j}$. The damage arising from pollution is uncertain, subject to a risk $\tilde{\theta}_{i}>0$. We focus here on situations where individual risks are ex-ante identical. ${ }^{11}$ For instance, damage may be the same for everybody, $\widetilde{\theta}_{i}=\widetilde{\theta}$ or individual damages $\widetilde{\theta}_{i}$ may be identically and independently distributed. ${ }^{12}$ This assumption allows us to write $\tilde{\theta}_{i}=\tilde{\theta}$ in our payoff formulation below. Let $\bar{\theta}$ denote the expected value of the risk $\tilde{\theta}$.

We make the following assumptions regarding the benefits and costs of pollution. First, the benefits are simply equal to $e_{i}$ in any state of nature. That is, benefits from polluting are not affected by uncertainty, and the individual level of pollution emission is identified with its monetary value. ${ }^{13}$ Second, the risk is multiplicative. There exists a damage function $d$ such that the individual damage from pollution is equal to $\tilde{\theta} d\left(\sum_{j=1}^{n} e_{j}\right)$. This function is increasing $d^{\prime}>0$, convex $d^{\prime \prime} \geq 0$ and satisfies $d(0)=0, d^{\prime}(0)<1 / \bar{\theta}$, and $\lim _{e \rightarrow+\infty} d^{\prime}(e)=+\infty$. Finally, agents have identical risk preferences represented by a von Neumann-Morgenstern utility function $u$, which is increasing $u^{\prime}>0$ and concave $u^{\prime \prime} \leq 0$. Agent $i$ 's expected utility is thus equal to

$$
E_{\tilde{\theta}} u\left(e_{i}-\tilde{\theta} d\left(\sum_{j=1}^{n} e_{j}\right)\right)
$$

The remainder of the paper is devoted to the analysis of this game. We focus on pure strategies, and will notably look at welfare in equilibrium. We adopt a simple approach. Since our game is

\footnotetext{
${ }^{11}$ This assumption is relaxed in section IV.

${ }^{12}$ Another example is when one and only one individual can be affected by the total damage, but this individual is unknown ex-ante.

${ }^{13}$ We consider uncertain benefits in section $\mathrm{V}$.
} 
symmetric, we define welfare as the sum of the expected utilities of the agents. For a symmetric profile where everyone pollutes $e$, this yields:

$$
W(e)=n E_{\tilde{\theta}} u(e-\tilde{\theta} d(n e))
$$

Our assumptions imply that welfare is a concave function of $e$, and that expected utility (II.1) is a concave function of $e_{i}$. Thus, an interior symmetric Nash equilibrium where everyone emits $e$ is characterized by the following equation:

$$
E_{\tilde{\theta}}\left(1-\tilde{\theta} d^{\prime}(n e)\right) u^{\prime}(e-\tilde{\theta} d(n e))=0
$$

At the equilibrium, agents set their emissions to equalize their expected marginal benefits from pollution $E_{\tilde{\theta}} u^{\prime}(e-\tilde{\theta} d(n e))$ to their expected private marginal damage $E_{\tilde{\theta}} \tilde{\theta} d^{\prime}(n e) u^{\prime}(e-\tilde{\theta} d(n e))$. They do not account for the effect of their emissions on others. Private marginal damage is $n$ times lower than social marginal damage, and there is overpollution at the equilibrium.

We next study equilibrium existence, symmetry, and unicity. Our findings will allow us to focus on symmetric interior Nash equilibria in our main analysis. ${ }^{14}$ We first show that existence is indeed guaranteed.

Proposition 1. An interior symmetric equilibrium always exists.

This result relies on the properties of marginal damage at extreme values. Since marginal damage is relatively low at low pollution levels, agents have some incentive to pollute. On the other hand marginal damage becomes arbitrarily large with pollution, hence the incentive to pollute is bounded. We next look at the shape of equilibria. The game being symmetric, we expect equilibria to be symmetric as well, although this is by no means guaranteed. Our next result shows that the outcome actually depends on the curvature of the utility function. Recall, utility function $u$ satisfies Decreasing (Constant, Increasing) Absolute Risk Aversion, or DARA (CARA, IARA), if the level of risk aversion $-u^{\prime \prime} / u^{\prime}$ is decreasing (constant, increasing).

Proposition 2. When u satisfies DARA or IARA, any interior equilibrium is symmetric. When u satisfies CARA, asymmetric equilibria exist.

\footnotetext{
${ }^{14}$ An equilibrium $\left(e_{1}, \ldots, e_{n}\right)$ is interior if $\forall i, e_{i}>0$.
} 
Interior equilibria are indeed symmetric when the level of risk aversion is monotonic. This does not hold for CARA utility functions, however. Due to the absence of income effect, the equilibrium conditions under CARA turn out to be invariant to redistributions of global pollution across agents. Under CARA, given some equilibrium, any profile with the same level of overall pollution is also an equilibrium. To prove Proposition 2, we apply the diffidence theorem on appropriate reformulations of the equilibrium conditions (Gollier, 2001). We make use of similar techniques to prove our third result on unicity.

Proposition 3. When $u$ satisfies DARA or CARA, there is a unique symmetric equilibrium.

Taken together, these results imply that when the utility function is DARA there exists a unique interior equilibrium, which is symmetric. As a consequence, we focus on symmetric equilibria in what follows. We assume that equation (II.2) has a unique solution, that we denote by $e^{N}$.

\section{Effects of uncertainty and risk aversion}

\section{A. Comparison with certainty and risk neutrality}

Our main objectives is to study the impact of uncertainty and risk aversion on emissions and welfare in equilibrium. We first look at the natural benchmarks of certainty and risk neutrality. In our model, the curvature of $u$ only captures risk aversion motives. Uncertainty does not affect risk neutral agents' emissions and the emissions of risk averse agents under certainty are identical to the emissions of risk neutral agents. When damage is certain or when agents are risk neutral, condition (II.2) becomes

$$
1-\bar{\theta} d^{\prime}(n e)=0
$$

Denote by $\bar{e}^{N}$ the corresponding emission level. Our next result compares this benchmark with the general case of uncertainty.

Proposition 4. Emissions under uncertainty are lower than under certainty: $e^{N} \leq \bar{e}^{N}$.

Consistent with the remark above, this proposition also implies that emissions are lower under risk aversion than under risk neutrality. We provide some intuition for this result. Consider a 
change from $\bar{\theta}$ to $\tilde{\theta}$. Suppose first that others' emissions do not change: $\sum_{j \neq i} e_{j}=(n-1) \bar{e}^{N}$. Under uncertainty, agent $i$ 's payoff is equal to:

$$
\pi_{i}=e_{i}-\tilde{\theta} d\left(e_{i}+(n-1) \bar{e}^{N}\right)
$$

Agent $i$ should emit $\bar{e}^{N}$ if he wants to maximize the expected value of his payoff $E_{\tilde{\theta}} \pi_{i}=e_{i}-\bar{\theta} d\left(e_{i}+\right.$ $\left.(n-1) \bar{e}^{N}\right)$. Thus, decreasing $e_{i}$ with respect to this benchmark leads to a reduction in expected payoffs. On the other hand, it also reduces the payoff's variance $\operatorname{var}\left(\pi_{i}\right)=\operatorname{var}(\tilde{\theta}) d^{2}\left(e_{i}+(n-1) \bar{e}^{N}\right)$. Lowering emissions acts as a form of insurance here. Assuming others' emissions are fixed, reducing emissions trades-off a loss in expected payoff for a reduction in risk. The emissions of others are not fixed, however. They care about uncertainty, and also react to each other's actions. Strategic effects could especially lead some agents to increase their own emissions as a response to a decrease in others' emissions. In our setting, we are able to show that these strategic effects are dominated. The reason is that the whole best-response functions are lower under uncertainty. Thus, their intersection with the 45 degree line, which determines the symmetric equilibrium, is also lower.

A key implication of this result is that welfare may be higher under uncertainty. To see why, denote by $\bar{W}(e)=n u(e-\bar{\theta} d(n e))$ welfare under certainty. Compare the levels of welfare in equilibrium under uncertainty $W\left(e^{N}\right)$ and under certainty $\bar{W}\left(\bar{e}^{N}\right)$. Their difference is the sum of two terms:

$$
W\left(e^{N}\right)-\bar{W}\left(\bar{e}^{N}\right)=\left[W\left(e^{N}\right)-W\left(\bar{e}^{N}\right)\right]+\left[W\left(\bar{e}^{N}\right)-\bar{W}\left(\bar{e}^{N}\right)\right]
$$

Uncertainty has two effects, as captured by the two bracketed terms of the right hand side of this equality. It leads to a decrease in emissions, holding the risk fixed (first term), and it changes the risk faced, holding pollution emissions fixed (second term). The first term represents the strategic effect and is positive by Proposition 4, concavity of $W$, and the fact that $W^{\prime}\left(e^{N}\right)<0 .{ }^{15}$ The second term represents the risk aversion effect and is negative by concavity of $u$ and Jensen's inequality. The overall effect is ambiguous. Uncertainty may be socially beneficial, if the indirect positive effect of reduced emissions is greater than the direct negative effect of uncertainty.

\footnotetext{
${ }^{15}$ Using condition (II.2), it is easy to see that $W^{\prime}\left(e^{N}\right)=-n(n-1) d^{\prime}\left(n e^{N}\right) E_{\tilde{\theta}} \tilde{\theta} u^{\prime}\left(e^{N}-\tilde{\theta} d\left(n e^{N}\right)\right)<0$.
} 
We next obtain conditions under which uncertainty yields higher welfare for small risks. Suppose here that $\tilde{\theta}=\bar{\theta}+k \tilde{\varepsilon}$, with $E \tilde{\varepsilon}=0$. A small risk corresponds to a small value of $k$. Introduce an indice $I_{d}$ measuring variations in the curvature of the damage function.

$$
I_{d}=\left(\frac{d}{d^{\prime}}\right)^{\prime}
$$

Using Taylor approximations, we can show that this indice determines the effect of uncertainty on welfare for small risks.

Proposition 5. If $I_{d}>(<)-\frac{n-2}{n}$ and the risk is small enough, uncertainty increases (decreases) welfare compared to certainty, $W\left(e^{N}\right)>(<) \bar{W}\left(\bar{e}^{N}\right)$.

Welfare is thus higher under uncertainty for any small enough risk and for any risk averse utility, as soon as $I_{d}$ is not too negative. This condition is always satisfied if, for instance, $d$ has constant elasticity. ${ }^{16}$ Interestingly, the condition does not depend on the shape of $u$, as long as $u$ is increasing and concave. Computations in the Appendix make clear, however, that the magnitude of the effects is affected by $u$. Another noteworthy feature of Proposition 5 is that a higher $n$ makes a positive effect of uncertainty on welfare more likely. An increase in the number of agents exacerbates free-riding, which increases the relative importance of the decrease in emissions in the welfare comparison.

Overall, this section showed that emissions are lower under uncertainty than under certainty, and that welfare may be higher. In the next two sections, we try to go further than this benchmark comparison.

\section{B. Increase in risk}

We first look at the effect of an increase in risk. That is, we compare outcomes for a risk $\tilde{\theta}$ and for a risk $\tilde{z}$ that is more uncertain in the sense of Rothschild and Stiglitz (1970). To emphasize this focus in what follows, we denote by $e^{N}(\widetilde{\theta})$ the emission level at the symmetric equilibrium under the risk $\tilde{\theta}$. Comparative statics with respect to the level of risk turn out to be ambiguous and to involve properties of the third derivative of the utility function. ${ }^{17}$ We present three partial results

\footnotetext{
${ }^{16}$ Suppose that $d(e)=d_{0} e^{\alpha}$ with $\alpha \geq 1$. Then $I_{d}=1 / \alpha>0$.

${ }^{17}$ These features are consistent with earlier findings in models with a single decision maker (Hadar and Seo, 1990; Gollier, 2001).
} 
here. First, we provide an informal analysis of what happens for small damages. This illustrates the emergence of effects related to prudence that tend to increase emissions. Second, we show that an increase in risk always leads to a decrease in emissions in the absence of prudence. Third, we show that an increase in risk also leads to a decrease in emissions for a natural class of binary risks.

Suppose first that $d$ is small. We can approximate agent $i$ 's utility as follows.

$$
u\left(e_{i}-\theta d\left(e_{i}+e_{-i}\right)\right) \approx u\left(e_{i}\right)-\theta d\left(e_{i}+e_{-i}\right) u^{\prime}\left(e_{i}\right)+\frac{1}{2} \theta^{2} d\left(e_{i}+e_{-i}\right)^{2} u^{\prime \prime}\left(e_{i}\right)
$$

Rewrite equation (II.2) as $E_{\tilde{\theta}} f(e, \tilde{\theta})=0$ and consider an increase in risk from $\tilde{\theta}$ to $\tilde{z}$. The previous approximation leads to:

$$
E_{\tilde{z}} f(e, \tilde{z})-E_{\tilde{\theta}} f(e, \tilde{\theta})=\frac{1}{2}\left(E_{\tilde{z}} \tilde{z}^{2}-E_{\tilde{\theta}} \tilde{\theta}^{2}\right)\left[2 d(n e) d^{\prime}(n e) u^{\prime \prime}(e)+d^{2}(n e) u^{\prime \prime \prime}(e)\right]
$$

Since $E_{\tilde{z}} \tilde{z}^{2} \geq E_{\tilde{\theta}} \tilde{\theta}^{2}$, the effect of an increase in risk on emissions is controlled by the sign of $2 d(n e) d^{\prime}(n e) u^{\prime \prime}(e)+d^{2}(n e) u^{\prime \prime \prime}(e)$. If this sign is negative, $E_{\tilde{z}} f(e, \tilde{z})$ lies everywhere below $E_{\tilde{\theta}} f(e, \tilde{\theta})$, which means that $e^{N}(\tilde{z}) \leq e^{N}(\tilde{\theta})$. The opposite happens if the sign is positive. This expression is the sum of two terms. The first term $2 d(n e) d^{\prime}(n e) u^{\prime \prime}(e)$ is always negative under risk aversion. This part captures the effect highlighted in Proposition 4. Greater uncertainty increases the damage's variance. To compensate, risk averse agents have an incentive to reduce their emissions. In contrast, the second term $d^{2}(n e) u^{\prime \prime \prime}(e)$ represents a new effect. Following Kimball (1990), agents are said to be prudent when $u^{\prime \prime \prime} \geq 0$. Under prudence, the marginal value of an extra unit of emissions $e$ is higher when uncertainty is greater. ${ }^{18}$ Prudent agents have thus an incentive to increase their emissions when they face more uncertainty. By polluting more, agents are able to increase the risk-free portion of their payoff. Overall, the impact is ambiguous and depends on which effect dominates. This opposition between risk aversion and prudence motives holds more generally.

Our next result confirms, albeit negatively, the role played by prudence. We show that in the absence of prudence, emissions always decrease when the level of risk increases.

\footnotetext{
${ }^{18}$ Formally, prudence implies that $E_{\tilde{z}} u^{\prime}(e-\tilde{z} d(n e)) \geq E_{\tilde{\theta}} u^{\prime}(e-\tilde{\theta} d(n e))$.
} 
Proposition 6. Consider an increase in risk from $\tilde{\theta}$ to $\tilde{z}$. Suppose that $u^{\prime \prime \prime}=0$. Then, for any damage function $d$, an increase in risk leads to lower emissions, $e^{N}(\tilde{z}) \leq e^{N}(\tilde{\theta})$.

There are two ways to obtain non-ambiguous comparative statics here. First, as done in the previous result, we can restrict preferences and look for results valid for any increase in risk. Alternatively, we can study specific risks, and look for results valid for any risk averse utility functions. We illustrate this approach next. We introduce and define catastrophic risks as follows. Damages may only be low or high (a catastrophe occurs). We denote by $\theta_{L}$ the risk associated with low damages, by $\bar{\theta}$ the expected value of the risk, and by $1 / k$ (with $k>1$ ) the catastrophe's probability. Thus, $\tilde{\theta}$ is equal to $\theta_{L}$ with probability $1-\frac{1}{k}$ and to $\theta_{L}+k\left(\bar{\theta}-\theta_{L}\right)$ with probability $1 / k$. We define an increase in catastrophic risk as an increase in $k$, holding $\theta_{L}$ and $\bar{\theta}$ constant. An increase in catastrophic risk is a particular case of Rothschild and Stiglitz's increase in uncertainty. As $k$ increases, the catastrophe becomes less probable but more damaging. We obtain the following result.

Proposition 7. For any utility function $u$ and damage function $d$, an increase in catastrophic risk leads to lower emissions.

Thus, prudence effects are dominated for catastrophic risks. In this case, as in all cases where an increase in risk leads to lower emissions, our previous welfare analysis carries over. ${ }^{19} \mathrm{An}$ increase in risk may increase welfare if the indirect positive effect of reduced emissions is greater than the direct negative effect. We present some numerical results to further illustrate these effects. In our simulations, we assume that the utility function satisfies Constant Relative Risk Aversion: $u(\pi)=\left(\pi_{0}+\pi\right)^{1-\gamma} /(1-\gamma)$ if $\gamma \neq 1$ and $\ln \left(\pi_{0}+\pi\right)$ if $\gamma=1$ with $\pi_{0}=10$. Damage is quadratic $d(e)=\frac{1}{2} e^{2}$. The risk is catastrophic, with $\theta_{L}=1$ and $\bar{\theta}=2$. We consider increases in $k$ ranging from 2 to 82 by increments of 10 . We suppose that $n=2$ and look at three values for the coefficient of relative risk aversion: $\gamma=1 / 2,1$, and 2. Figure 1 describes the level of emission in equilibrium as a function of the level of risk. Observe that here emission under certainty is equal to 0.25 . We see that emission is always lower than under certainty, and decreases as the level of risk increases, which is consistent with Propositions 4 and 7. Emission is also lower when risk

\footnotetext{
${ }^{19}$ In contrast, if an increase in risk leads to higher emissions, the direct and the indirect effects go in the same direction, and welfare decreases.
} 
aversion is higher. We show in the next section that this holds more generally. More importantly, Figure 2 describes how welfare varies with the level of risk. We examine the percentage difference relative to welfare under certainty. This number is always positive in the simulations, implying that welfare under uncertainty is greater than under certainty. This shows that Proposition 5 does not merely follow from the assumption of a small risk. In addition, an increase in catastrophic risk always leads to an increase in welfare in these simulations. The positive effect of reduced emissions dominates the negative effect of an increased risk.

\section{Insert Figures 1 and 2 about here}

\section{Increase in risk aversion}

We next study how a change in the level of risk aversion affects emissions. Holding the risk constant, we look at the effect of an Arrow-Pratt increase in risk aversion. Agents with utility function $v$ are more risk averse than those with utility $u$ if there exists a function $\Phi$ satisfying $\Phi^{\prime}>0$ and $\Phi^{\prime \prime} \leq 0$ and such that $v=\Phi(u)$ (Pratt, 1964). Do more risk averse agents emit less pollution? Our next result shows that the answer is affirmative. For clarity, we denote here by $e^{N}(u)$ the emission level at the symmetric equilibrium when agents have utility function $u$.

Proposition 8. Suppose that agents with utility $v$ are more risk averse than those with utility $u$. Then, an increase in risk aversion leads to lower emissions, $e^{N}(v) \leq e^{N}(u)$.

The intuition behind Proposition 4 applies here as well. Holding others' emissions constant, lowering emissions leads to a reduction in the payoff's variability. Such a reduction is more and more desirable as risk aversion increases. And while others' emissions are not fixed and strategic effects come into play, they are never strong enough to overcome the direct effect. Thus, emissions in equilibrium decrease if the level of risk aversion increases, which directly generalizes the benchmark comparison between risk aversion and risk neutrality (Proposition 4). This stands in contrast with the effect of an increase in risk. ${ }^{20}$

\footnotetext{
${ }^{20}$ As agents become more risk-averse, they might also become more prudent. The previous section suggests that this could lead to an increase in emissions. Our result shows that this never happens. Here, the negative effect on emissions due to more risk-aversion is always greater than potentially positive effect due to more prudence.
} 


\section{Risk Heterogeneity}

In this section, we relax the assumption that agents are homogeneous. As we focus on the effect of uncertainty, we only consider heterogeneity with respect to the level of risk faced. As we will show, risk heterogeneity has a drastic effect on equilibrium properties and results. To see this, we simply consider an economy with two agents. One agent faces uncertain damage $\widetilde{\theta}$ while the other agent faces certain damage $\bar{\theta}=E \widetilde{\theta}$. We especially ask: Which agent is going to emit the most? Is uncertainty detrimental or an advantage?

Let agent 1 be the agent who faces no risk, and agent 2 the one who faces a risk. We first show that the equilibrium cannot be interior. Consider the conditions

$$
\begin{aligned}
1-\bar{\theta} d^{\prime}\left(e_{1}^{N}+e_{2}^{N}\right) & =0 \\
E\left(1-\widetilde{\theta} d^{\prime}\left(e_{1}^{N}+e_{2}^{N}\right)\right) u^{\prime}\left(e_{2}^{N}-\widetilde{\theta} d\left(e_{1}^{N}+e_{2}^{N}\right)\right) & =0,
\end{aligned}
$$

where $e_{1}^{N}$ is the equilibrium level of emissions of agent 1 and $e_{2}^{N}$ that of agent 2. Through (IV.1), we obtain

$$
E\left(1-\widetilde{\theta} d^{\prime}\left(e_{1}^{N}+e_{2}^{N}\right)\right) u^{\prime}\left(e_{2}^{N}-\widetilde{\theta} d\left(e_{1}^{N}+e_{2}^{N}\right)\right)=\operatorname{cov}\left[1-\widetilde{\theta} d^{\prime}\left(e_{1}^{N}+e_{2}^{N}\right), u^{\prime}\left(e_{2}^{N}-\widetilde{\theta} d\left(e_{1}^{N}+e_{2}^{N}\right)\right)\right]
$$

This covariance is strictly negative when $u$ is strictly concave, which contradicts (IV.2). This means that, in equilibrium, one agent must emit no emissions at all. We show that it can only be agent 2 , and that this indeed constitutes an equilibrium.

Proposition 9. Consider an economy in which agent 1 faces no risk and agent 2 faces a risk. There is a unique equilibrium in which agent 2 does not emit, and agent 1 emits $e$ which solves $1-\bar{\theta} d^{\prime}(e)=0$.

This result is caused by strong strategic effects. Its intuition may be presented as follows. Start with a situation where both agents face no uncertainty. Suppose then that agent 2 faces some degree of uncertainty about his own damage. This leads agent 2 to reduce his emissions, for a given level of emissions of agent 1. Since emissions are perfect strategic substitutes under certainty, this leads agent 1 to increase his own emissions. This increase in emissions of agent 1 gives a further incentive to agent 2 to reduce his emissions, and so on. The process continues 
until agent 2 emits no more and agent 1 emits as much as if he were alone in the economy.

Note also that agent 1's level of emissions is actually equal to the total emissions obtained in equilibrium under certainty, and that total pollution is the same in the economy. Therefore, it is immediate that agent 1 is better-off compared to the full certainty case and that agent 2 is worse-off. This raises the question of whether the increase in agent 1's expected utility may compensate agent 2's decrease. That is, how does heterogeneous uncertainty affect welfare in equilibrium compared to certainty? It is actually easy to see that it always reduces welfare.

Proposition 10. Consider an economy in which agent 1 faces no risk and agent 2 faces a risk. Then, welfare is lower than when both agents face no risk.

This result suggests that risk never has a positive effect on welfare in this economy, unlike in the symmetric economy. Notice however that, starting with the same heterogenous economy, adding a risk to agent 1 can increase welfare. This is the consequence of Proposition 5, which together with the last Proposition implies that the effect of adding individual risks on welfare may be non-monotonic.

Finally, we want to contrast non-cooperative and cooperative emissions. ${ }^{21}$ For the sake of comparison, social welfare is still defined as the sum of both agents' expected utilities. Thus, the cooperative levels of emission solve the following maximization program

$$
\max _{e_{1}, e_{2}} u\left(e_{1}-\bar{\theta} d\left(e_{1}+e_{2}\right)\right)+E_{\tilde{\theta}} u\left(e_{2}-\tilde{\theta} d\left(e_{1}+e_{2}\right)\right)
$$

We find that, under prudence, agent 2 emits more than agent 1 at the optimum. The intuition for this result is simple. Under prudence, agent 2 values more an extra unit of emissions than agent 1 . Allowing him to emit more is therefore socially efficient.

How does uncertainty affect welfare? Since the objective is concave in the risk variable $\widetilde{\theta}$, uncertainty clearly reduces aggregate expected utilities compared to the certainty case. We further question the distribution of this welfare reduction. Does agent 1 end up with more or less utility than agent 2 ? The answer depends again on the shape of the utility function. Indeed, we

\footnotetext{
${ }^{21}$ Properties of the cooperative solution are throroughly analyzed in our working paper, see Bramoullé and Treich (2004). In the symmetric case, results on the effects of uncertainty under cooperation are similar to those obtained under non-cooperation. Especially, cooperative emissions are lower under uncertainty than under certainty. However, they differ on welfare, the effect of an increase in risk, and heterogenous risks. The last situation is discussed in this section.
} 
can show that, under DARA, agent 2 has a higher expected utility than agent 1 at the cooperative emissions level. The proof relies on the fact that, under DARA, $-u^{\prime}$ is more concave than $u$. As a result, the increase in emissions that makes agent 2's marginal utility equal to agent 1's marginal utility is larger than the increase in emissions needed to equate both expected utilities. All in all, these results suggest that, under the usual DARA assumption (which implies prudence), facing uncertainty may be viewed as an advantage under cooperation.

In this section, we have shown that when agents are heterogeneous in the risk they face, the impact of uncertainty on individual expected utility goes in opposite directions in the cooperative and the non-cooperative case. Facing uncertainty is generally beneficial under cooperation. In contrast, facing uncertainty is always detrimental under non-cooperation. In both cases, uncertainty reduces aggregate expected utility.

\section{Extensions}

In this section, we discuss the robustness of our results to different formulations of the model. Our global commons model depends on three key variables: individuals' emissions $e$, total emissions (hereafter denoted by) $\Sigma$, and a risk parameter $\theta$. Payoffs in a general model can thus be written $v(e, \Sigma, \theta)$. So far, we have studied the case where $v(e, \Sigma, \theta)=u(e-\theta d(\Sigma)) .{ }^{22}$ We now consider three alternative formulations:

- an additive risk model $v(e, \Sigma, \theta)=u(e-d(\Sigma)+\theta)$

- an uncertain benefit model $v(e, \Sigma, \theta)=u(\theta e-d(\Sigma))$;

- and a two-period model $v(e, \Sigma, \theta)=u_{0}\left(w_{0}+e\right)+u_{1}\left(w_{1}-\theta d(\Sigma)\right)$ with $u_{0}$ and $u_{1}$ increasing and concave.

We examine each model in turn, focusing on the comparison between uncertainty and certainty in the homogenous case. We consider first the simple additive risk model $v(e, \Sigma, \theta)=u(e-d(\Sigma)+$

\footnotetext{
${ }^{22}$ We observe that many papers in the climate change literature have considered a per period utility of the form $v(e, \Sigma, \theta)=u(e)-\theta d(\Sigma)$; see e.g., Ulph and Ulph (1996), Ulph and Maddison (1997), and Baker (2005). Notice though that, in this case, uncertainty (unlike learning) has no effect on decisions and payoffs because the utility is linear in $\theta$.
} 
$\theta)$. Here, the equilibrium condition under uncertainty writes:

$$
\left(1-d^{\prime}(n e)\right) E_{\tilde{\theta}} u^{\prime}(e-d(n e)+\tilde{\theta})=0
$$

Since $E_{\tilde{\theta}} u^{\prime}(e-d(n e)+\tilde{\theta})$ is strictly positive, this condition is the same as under certainty: $1-d^{\prime}(n e)=0$. The additive risk does not affect emissions. Therefore, there is no positive indirect effect and uncertainty always has a negative impact on welfare in this economy.

Next, we look at the model with uncertain benefits $v(e, \Sigma, \theta)=u(\theta e-d(\Sigma))$. The equilibrium condition under uncertainty becomes:

$$
E_{\tilde{\theta}}\left(\tilde{\theta}-d^{\prime}(n e)\right) u^{\prime}(\tilde{\theta} e-d(n e))=0 .
$$

We analyze this condition in the Appendix, and show that emissions are still lower under uncertainty than under certainty. The argument behind Proposition 4 essentially extends. When benefits are uncertain, the payoff variance is still increasing in emissions. Hence, a decrease in emissions is still desirable under risk-aversion. We also generalize our analysis of small risks to this case. We obtain a result similar to Proposition 5. For any risk small enough and any risk-averse utility function, uncertainty has a positive impact on welfare if the damage function satisfies a simple curvature condition. We check that this condition is indeed satisfied in special cases. Thus, even with uncertain benefits, uncertainty lowers emissions and may increase welfare.

Finally, we study the two-period model $v(e, \Sigma, \theta)=u_{0}\left(w_{0}+e\right)+u_{1}\left(w_{1}-\theta d(\Sigma)\right)$. The equilibrium condition under uncertainty now becomes:

$$
u_{0}^{\prime}\left(w_{0}+e\right)-d^{\prime}(n e) E_{\tilde{\theta}} \tilde{\theta} u_{1}^{\prime}\left(w_{1}-\tilde{\theta} d(n e)\right)=0 .
$$

Interestingly, risk-aversion is not sufficient to sign the effect of uncertainty. We show in the Appendix that under risk-aversion and prudence, emissions under uncertainty are lower than under certainty. Prudence appears because in this model decreasing emissions leads to a form of precautionary savings. This is different from the effect highlighted in section III. In general, prudence expresses an incentive to secure a higher expected payoff in the period where the risk is faced. Here, a reduction in payoffs in the first, risk-free period leads to an increase in expected 
payoffs and to a decrease in payoff variance in the second period. Prudence and risk-aversion go in the same direction. In contrast, there is a unique period in the analysis of section III. Increasing expected payoffs may be then done by increasing emissions, but at the cost of increasing the variance. Prudence and risk-aversion go in opposite directions, and risk-aversion often dominates, as we showed. We generalize the analysis for small risks for the two-period model as well, and find that welfare may indeed be higher under uncertainty due to the positive effect of reduced emissions.

Our discussion so far has left aside two important features of global commons problems: the heterogeneity of impacts and dynamic issues. Would our results be robust to the introduction of these two features? We informally discuss them in turn. Consider first heterogeneity. As illustrated in section IV, our model could easily be generalized to account for different benefits, costs, risks, and levels of risk-aversion. In the presence of heterogeneity, best-response functions are still lower under uncertainty than under certainty. While a decrease in overall pollution is not guaranteed any more, it will certainly hold under specific conditions, for instance if heterogeneity is not too large. And as soon as pollution decreases, welfare may rise. Thus, our results should extend, under appropriate restrictions, to a setting with heterogeneity.

Dynamic models introduce significant new issues. First, the link between emissions and pollution is more complicated. On the one hand, current emissions have relatively little impact on current $\mathrm{CO} 2$ concentrations. On the other hand, current emissions affect $\mathrm{CO} 2$ concentrations in many future periods. We expect uncertainty to reduce current emissions, as long as emissions increase the variability of future damages and the discount rate is not too high. Second, uncertainty may affect damages through irreversibilities and thresholds. If anything, serious threats of irreversible changes should lead countries to increase their efforts to curb emissions, even in the absence of cooperation. Third, learning takes place over time. Much future research is needed to precisely understand the effects of uncertainty, learning, and heterogeneity in a dynamic model of negative externality.

\section{Conclusion}

Free-riding and uncertainty are both primary concerns for environmental issues. In this paper, we develop a simple model with strategic interactions among polluters and where the damage 
from pollution is subject to a multiplicative risk. We find that uncertainty often alleviates the negative externality. Polluters may lower their emissions to reduce their exposure to risk, which can increase overall welfare.

Our analysis may have interesting implications for the way economists traditionally view insurance and risk-sharing institutions. Usually, risk-sharing opportunities operate as a pure riskreduction mechanism, which improves welfare under risk-aversion. However, our paper suggests that this common risk-reduction may lead polluters to increase their emissions, and the net impact of risk-sharing opportunities that may be made available to polluters can be overall negative. A policy implication is that it may not be desirable to establish risk-sharing institutions before addressing a pollution problem.

Our analysis also sheds new light on the effect of uncertainty on the incentives to reach an agreement. A classical argument is that reaching an agreement may be easier under a "veil of uncertainty". ${ }^{23}$ In this view, cooperation is compared ex ante, before uncertainty is resolved, and ex post, once uncertainty is resolved. Cooperation is more likely to emerge ex ante, because more agents potentially gain from the agreement before the uncertainty is resolved. In contrast our results show that, from an ex ante perspective, cooperation may be less likely under uncertainty. The reason is that the difference in social welfare between cooperation and non-cooperation may be lower under uncertainty. This difference exactly measures the collective gain to reach an agreement. ${ }^{24}$ Hence, by partly alleviating the commons problem, uncertainty may also reduce the incentives to fully solve it.

\footnotetext{
${ }^{23}$ See Na and Shin (1998) for a formal analysis and Young (1994) for a general discussion.

${ }^{24}$ Welfare under cooperation drops under uncertainty. Thus, if welfare at equilibrium increases, the difference between the two decreases.
} 


\section{Appendix.}

The Appendix contains the proofs of all the Propositions in the paper, as well as sketches of the proofs of statements in sections IV and V. More detailed proofs are available under request. See also Bramoullé and Treich (2004).

Proof of Proposition 1: An interior symmetric equilibrium always exists.

Introduce $f(e, \theta)=\left(1-\theta d^{\prime}(n e)\right) u^{\prime}(e-\theta d(n e))$ and $\varphi(e)=E_{\tilde{\theta}} f(e, \tilde{\theta})$. Symmetric equilibria correspond to the zeros of $\varphi$. Remember that $d^{\prime}(0)<1 / \bar{\theta}$ and $\lim _{e \rightarrow+\infty} d^{\prime}(e)=+\infty$. First, observe that $\varphi(0)=\left(1-\bar{\theta} d^{\prime}(0)\right) u^{\prime}(0)>0$. Next we show that $\varphi(e)<0$ if $e$ is large enough. To simplify, suppose that there exists $\underline{\theta}$ such that $\tilde{\theta} \geq \underline{\theta}>0$. In that case, we have $f(e, \theta) \leq$ $\left(1-\underline{\theta} d^{\prime}(n e)\right) u^{\prime}(e-\theta d(n e))$ for any $\theta$, and thus $\varphi(e) \leq\left(1-\underline{\theta} d^{\prime}(n e)\right) E u^{\prime}(e-\tilde{\theta} d(n e))$. Then, notice that the right hand side of the last expression can be made negative for $e$ large enough. This argument essentially extends to the case where $\inf (\tilde{\theta})=0$. We can show that for $\varepsilon$ small enough and $e$ large enough, $E_{\tilde{\theta}<\varepsilon} f(e, \tilde{\theta})$ is dominated by $E_{\tilde{\theta} \geq \varepsilon} f(e, \tilde{\theta})$ and $\varphi(e)<0$. In the end, continuity ensures the existence of a zero of $\varphi$.QED.

Proof of Proposition 2: When u satisfies DARA or IARA, any interior equilibrium is symmetric. When u satisfies CARA, asymmetric equilibria exist.

Consider $\left(e_{1}^{*}, \ldots, e_{n}^{*}\right)$ an interior equilibrium. Introduce $e^{*}=\sum_{i=1}^{n} e_{i}^{*}, g(e, \theta)=\left(1-\theta d^{\prime}\left(e^{*}\right)\right) u^{\prime}(e-$ $\left.\theta d\left(e^{*}\right)\right)$, and $G(e)=E_{\tilde{\theta}} g(e, \tilde{\theta})$. Equilibrium conditions become: $\forall i, G\left(e_{i}^{*}\right)=0$. We will show that the equation $G(e)=0$ cannot have multiple solutions. We first prove the following useful property.

Lemma 1. If $u$ satisfies DARA, then $\forall e \geq 0, \exists \lambda>0: \forall \theta \geq 0, \frac{\partial g}{\partial e}(e, \theta) \geq-\lambda g(e, \theta)$ with a strict inequality except on a single value of $\theta$.

Proof: Introduce $\theta_{0}=1 / d^{\prime}\left(e^{*}\right)$ and $\lambda=A\left(e-\theta_{0} d\left(e^{*}\right)\right)$ where $A(\pi)=-u^{\prime \prime}(\pi) / u^{\prime}(\pi)$. If $\theta>$ $\theta_{0}$, then $1-\theta d^{\prime}\left(e^{*}\right)<0$ and $A\left(e-\theta d\left(e^{*}\right)\right)>\lambda$ since $A$ is decreasing and $d\left(e^{*}\right)>0$. Thus, $u^{\prime \prime}\left(e-\theta d\left(e^{*}\right)\right)<-\lambda u^{\prime}\left(e-\theta d\left(e^{*}\right)\right)$ and $\left(1-\theta d^{\prime}\left(e^{*}\right)\right) u^{\prime \prime}\left(e-\theta d\left(e^{*}\right)\right)>-\lambda\left(1-\theta d^{\prime}\left(e^{*}\right)\right) u^{\prime}\left(e-\theta d\left(e^{*}\right)\right)$. If $\theta<\theta_{0}$, then $\left(1-\theta d^{\prime}\left(e^{*}\right)\right)>0$ and $A\left(e-\theta d\left(e^{*}\right)\right)<\lambda$. This yields $u^{\prime \prime}\left(e-\theta d\left(e^{*}\right)\right)>-\lambda u^{\prime}\left(e-\theta d\left(e^{*}\right)\right)$ and, again, $\left(1-\theta d^{\prime}\left(e^{*}\right)\right) u^{\prime \prime}\left(e-\theta d\left(e^{*}\right)\right)>-\lambda\left(1-\theta d^{\prime}\left(e^{*}\right)\right) u^{\prime}\left(e-\theta d\left(e^{*}\right)\right)$. This means that for any $\theta \neq \theta_{0}, \frac{\partial g}{\partial e}(e, \theta)>-\lambda \varphi(e, \theta)$, while equality holds at $\theta=\theta_{0}$.

Next, we apply the diffidence theorem to function $g$, see Gollier (2001, p. 83). The previous lemma implies that $E_{\tilde{\theta}} g(e, \tilde{\theta})=0 \Rightarrow E_{\tilde{\theta}} \frac{\partial g}{\partial e}(e, \tilde{\theta})>0$. Thus, $G(e)=0 \Rightarrow G^{\prime}(e)>0$. This is 
a standard single-crossing property. It says that the function $G$ can only cross the horizontal axis from below, and ensures that it cannot cross it more than once. Therefore, any interior equilibrium is symmetric under DARA. When $u$ satisfies IARA, the previous reasoning can easily be adapted to show that $G(e)=0 \Rightarrow G^{\prime}(e)<0$. Function $G$ can only cross the horizontal axis from above, which also guarantees that the equation $G(e)=0$ cannot have multiple solutions.

Finally, suppose that $u$ satisfies CARA, $u(\pi)=-e^{-A \pi}$ and let $\left(e_{1}, \ldots, e_{n}\right)$ be a profile such that $\sum_{i=1}^{n} e_{i}=e^{*}$. Equilibrium conditions become: $\forall i, E_{\tilde{\theta}}\left(1-\tilde{\theta} d^{\prime}\left(e^{*}\right)\right) e^{-A\left(e_{i}^{*}-\tilde{\theta} d\left(e^{*}\right)\right)}=0$. Multiplying by $e^{-A\left(e_{i}-e_{i}^{*}\right)}$, we obtain: $\forall i, E_{\tilde{\theta}}\left(1-\tilde{\theta} d^{\prime}\left(\sum_{i=1}^{n} e_{i}\right)\right) e^{-A\left(e_{i}-\tilde{\theta} d\left(\sum_{i=1}^{n} e_{i}\right)\right)}=0$, which shows that $\left(e_{1}, \ldots, e_{n}\right)$ is also an equilibrium. QED.

Proof of Proposition 3: When u satisfies DARA or CARA, there is a unique symmetric equilibrium.

A symmetric equilibrium where everyone emits $e$ is characterized by the equation $\varphi(e)=0$. We will show that this equation cannot have multiple solutions. As in the previous proof, we will rely on the diffidence theorem to show a single-crossing property. Omitting arguments for clarity, we have: $\frac{\partial f}{\partial e}=-n \theta d^{\prime \prime} u^{\prime}+\left(1-\theta d^{\prime}\right)\left(1-n \theta d^{\prime}\right) u^{\prime \prime}$. Introduce $h=\left(1-\theta d^{\prime}\right)\left(1-n \theta d^{\prime}\right) u^{\prime \prime}$ and $\theta^{*}=1 / d^{\prime}(n e)$. Observe that $f\left(e, \theta^{*}\right)=h\left(e, \theta^{*}\right)=0$. We wish to apply Corollary 1 in

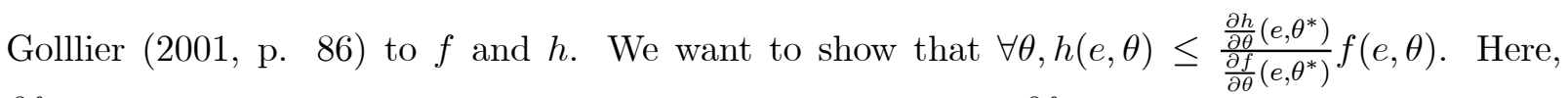
$\frac{\partial f}{\partial \theta}\left(e, \theta^{*}\right)=-d^{\prime}(n e) u^{\prime}\left(e-\theta^{*} d(n e)\right) \neq 0$ and $\frac{\partial h}{\partial \theta}\left(e, \theta^{*}\right)=-(n-1) \frac{\partial f}{\partial \theta}\left(e, \theta^{*}\right)$. The previous inequality is equivalent to

$$
\left(1-\theta d^{\prime}\right)(1-n) A\left(e-d / d^{\prime}\right) \leq\left(1-\theta d^{\prime}\right)\left(1-n \theta d^{\prime}\right) A(e-\theta d)
$$

Suppose first that $u$ satisfies CARA. $A$ is constant and this inequality reduces to $-(1-$ $\left.\theta d^{\prime}\right)^{2} n A \leq 0$, which is always satisfied. Suppose next that $u$ satisfies DARA, that is $A$ is decreasing. Consider 3 cases. (1) If $\theta \geq \theta^{*}$, then $A(e-\theta d) \geq A\left(e-d / d^{\prime}\right)$ and $\left(1-\theta d^{\prime}\right)\left(1-n \theta d^{\prime}\right) \geq 0$. Then, $\left(1-\theta d^{\prime}\right)\left(1-n \theta d^{\prime}\right) A(e-\theta d) \geq\left(1-\theta d^{\prime}\right)\left(1-n \theta d^{\prime}\right) A\left(e-d / d^{\prime}\right) \geq\left(1-\theta d^{\prime}\right)(1-n) A\left(e-d / d^{\prime}\right)$ where the second inequality comes from the result on CARA functions. (2) If $\theta^{*} \geq \theta \geq \frac{1}{n} \theta^{*}$, then $A(e-\theta d) \leq A\left(e-d / d^{\prime}\right)$ and $\left(1-\theta d^{\prime}\right)\left(1-n \theta d^{\prime}\right) \leq 0$, hence $\left(1-\theta d^{\prime}\right)\left(1-n \theta d^{\prime}\right) A(e-\theta d) \geq$ $\left(1-\theta d^{\prime}\right)(1-n) A\left(e-d / d^{\prime}\right)$ for the same reason. (3) If $\frac{1}{n} \theta^{*}>\theta$, then $1-\theta d^{\prime}>0$ and $1-n \theta d^{\prime}>0$ but $1-n<0$, hence the inequality is satisfied. 
Thus, under CARA or DARA, we can conclude that $\varphi(e)=E_{\tilde{\theta}} f(e, \tilde{\theta})=0 \Rightarrow E_{\tilde{\theta}} h(e, \tilde{\theta}) \leq$ $0 \Rightarrow \varphi^{\prime}(e)=E_{\tilde{\theta}} \frac{\partial f}{\partial e}(e, \tilde{\theta})<0$. This is a similar single-crossing condition as the one obtained in the previous proof. It means that $\varphi$ can only cross the horizontal axis once. Hence the symmetric equilibrium is unique. QED.

Proof of Proposition 4: Emissions under uncertainty are lower than under certainty: $e^{N} \leq \bar{e}^{N}$.

We make use of the following covariance rule, see Kimball (1951) or Gollier (2001, p. 94). If $X(\theta)$ is non-increasing in $\theta$ and $Y(\theta)$ is non-decreasing in $\theta$, then $\operatorname{cov}_{\tilde{\theta}}(X(\tilde{\theta}), Y(\tilde{\theta})) \leq 0$. Introduce $X(\theta)=1-\theta d^{\prime}\left(n e^{N}\right)$ and $Y(\theta)=u^{\prime}\left(e^{N}-\theta d\left(n e^{N}\right)\right) . \quad X$ is clearly non-increasing, and $Y$ is non-decreasing by concavity of $u$. This implies that $\operatorname{cov}_{\tilde{\theta}}(X(\tilde{\theta}), Y(\tilde{\theta})) \leq 0$. We have: $\operatorname{cov}_{\tilde{\theta}}(X(\tilde{\theta}), Y(\tilde{\theta}))=E X(\tilde{\theta}) Y(\tilde{\theta})-E X(\tilde{\theta}) E Y(\tilde{\theta})$. Then, $E X(\tilde{\theta}) Y(\tilde{\theta})=\varphi\left(e^{N}\right)=0$ and $E Y(\tilde{\theta})>0$. Therefore $\operatorname{EX}(\tilde{\theta})=1-\bar{\theta} d^{\prime}\left(n e^{N}\right) \geq 0$. Since the function $1-\bar{\theta} d^{\prime}(n e)$ is non-increasing in $e$ and $1-\bar{\theta} d^{\prime}\left(n \bar{e}^{N}\right)=0$, we obtain $e^{N} \leq \bar{e}^{N}$. QED.

Proof of Proposition 5: If $I_{d}>(<)-\frac{n-2}{n}$ and the risk is small enough, uncertainty increases (decreases) welfare compared to certainty, $W\left(e^{N}\right)>(<) \bar{W}\left(\bar{e}^{N}\right)$.

Denote by $e(k)$ the level of emissions at the symmetric equilibrium, by $W(k)$ the level of welfare and by $V$ the variance of $\tilde{\varepsilon}$. Note that $e(0)=\bar{e}^{N}$ and $\frac{\partial \tilde{\theta}}{\partial k}=\tilde{\varepsilon}$. Deriving condition (II.2) with respect to $k$ yields: $e^{\prime}(k) E \frac{\partial f}{\partial e}(e(k), \tilde{\theta})+E \tilde{\varepsilon} \frac{\partial f}{\partial \theta}(e(k), \tilde{\theta})=0$. Omitting arguments for clarity, we get: $\frac{\partial f}{\partial e}=-n \theta d^{\prime \prime} u^{\prime}+\left(1-\theta d^{\prime}\right)\left(1-n \theta d^{\prime}\right) u^{\prime \prime}$ and $\frac{\partial f}{\partial \theta}=-d^{\prime} u^{\prime}-d\left(1-\theta d^{\prime}\right) u^{\prime \prime}$. At $k=0$, this reduces to $E \frac{\partial f}{\partial e}=-n \bar{\theta} d^{\prime \prime} u^{\prime}<0$ and $E \tilde{\varepsilon} \frac{\partial f}{\partial \theta}=0$. Therefore, $e^{\prime}(0)=0$. Deriving a second time, we obtain: $e^{\prime \prime}(k) E \frac{\partial f}{\partial e}(e(k), \tilde{\theta})+e^{\prime}(k)()+.E \tilde{\varepsilon}^{2} \frac{\partial^{2} f}{\partial \theta^{2}}(e(k), \tilde{\theta})=0$. Since $\frac{\partial^{2} f}{\partial \theta^{2}}=2 d d^{\prime} u^{\prime \prime}+d^{2}\left(1-\theta d^{\prime}\right) u^{\prime \prime \prime}$, we have $E \tilde{\varepsilon}^{2} \frac{\partial^{2} f}{\partial \theta^{2}}=2 d d^{\prime} u^{\prime \prime} V$ at $k=0$. This means that

$$
e^{\prime \prime}(0)=\frac{2 d d^{\prime} u^{\prime \prime} V}{n \bar{\theta} d^{\prime \prime} u^{\prime}}
$$

The fact that $e^{\prime \prime}(0)<0$ is consistent with Proposition 4. Emissions for small risks are lower than for no risk. Next, derive $W$ with respect to $k$. Denote by $g(e, \theta)=u(e-\theta d(n e))$. $W^{\prime}(k)=$ $e^{\prime}(k) E \frac{\partial g}{\partial e}(e(k), \tilde{\theta})+E \tilde{\varepsilon} \frac{\partial g}{\partial \theta}(e(k), \tilde{\theta})$. Here, $\frac{\partial g}{\partial e}=\left(1-n \theta d^{\prime}\right) u^{\prime}$ and $\frac{\partial g}{\partial \theta}=-d u^{\prime}$. At $k=0$, we obtain, again, $W^{\prime}(0)=0$. Deriving a second time, $W^{\prime \prime}(k)=e^{\prime \prime}(k) E \frac{\partial g}{\partial e}(e(k), \tilde{\theta})+e^{\prime}(k)()+.E \tilde{\varepsilon}^{2} \frac{\partial^{2} g}{\partial \theta^{2}}(e(k), \tilde{\theta})$. 
Since $\frac{\partial^{2} g}{\partial \theta^{2}}=d^{2} u^{\prime \prime}$, this implies

$$
W^{\prime \prime}(0)=-e^{\prime \prime}(0)(n-1) u^{\prime}+d^{2} u^{\prime \prime} V
$$

We see, again, the two effects. The first term on the right hand side captures the positive strategic effect of a reduction in emissions on welfare. The second term is negative due to risk aversion. Substituting the expression for $e^{\prime \prime}(0)$ leads to $W^{\prime \prime}(0)=\left(-u^{\prime \prime}\right) V \frac{d d^{\prime 2}}{d^{\prime \prime}}\left(I_{d}+\frac{n-2}{n}\right)$. A Taylor approximation of $W$ around 0 yields $W(k) \approx W(0)+\frac{1}{2} k^{2} W^{\prime \prime}(0)+o\left(k^{2}\right)$. If $W^{\prime \prime}(0)>0$, $W(k)>W(0)$ if $k$ is small enough, while if $W^{\prime \prime}(0)<0, W(k)<W(0)$ if $k$ is small enough. QED.

Proof of Proposition 6: Consider an increase in risk from $\tilde{\theta}$ to $\tilde{z}$. Suppose that $u^{\prime \prime \prime}=0$. Then, for any damage function $d$, an increase in risk leads to lower emissions, $e^{N}(\tilde{z}) \leq e^{N}(\tilde{\theta})$.

Since $\tilde{z}$ corresponds to an increase in risk from $\tilde{\theta}$, for any concave function $\psi, E \psi(\tilde{z}) \leq E \psi(\tilde{\theta})$. If $f(e, \theta)$ is concave in $\theta$, then $\varphi(e, \tilde{z}) \leq \varphi(e, \tilde{\theta})$. We have:

$$
\frac{\partial^{2} f}{\partial \theta^{2}}=2 d(n e) d^{\prime}(n e) u^{\prime \prime}(e-\theta d(n e))+d^{2}(n e)\left(1-\theta d^{\prime}(n e)\right) u^{\prime \prime \prime}(e-\theta d(n e))
$$

hence $\frac{\partial^{2} f}{\partial \theta^{2}} \leq 0$ and $f$ concave if $u^{\prime \prime \prime}=0$. QED.

Proof of Proposition 7: For any utility function $u$ and damage function $d$, an increase in catastrophic risk leads to lower emissions.

Introduce $\pi_{L}=e-\theta_{L} d(n e)$ and $\pi_{H}=e-\left(\theta_{L}+k\left(\bar{\theta}-\theta_{L}\right)\right) d(n e)$. Condition II.2 becomes:

$$
\left(1-\frac{1}{k}\right)\left(1-\theta_{L} d^{\prime}(n e)\right) u^{\prime}\left(\pi_{L}\right)+\frac{1}{k}\left(1-\left(\theta_{L}+k\left(\bar{\theta}-\theta_{L}\right) d^{\prime}(n e)\right) u^{\prime}\left(\pi_{H}\right)=0\right.
$$

Define $\psi(e, k)=(k-1)\left(1-\theta_{L} d^{\prime}(n e)\right) u^{\prime}\left(\pi_{L}\right)+\left(1-\left(\theta_{L}+k\left(\bar{\theta}-\theta_{L}\right) d^{\prime}(n e)\right) u^{\prime}\left(\pi_{H}\right)\right.$. It is sufficient to show that $\psi$ is non-increasing in $k$. After some simplifications, we obtain

$$
\frac{\partial \psi}{\partial k}=\frac{u^{\prime}\left(\pi_{H}\right)}{1-k}\left(1-\bar{\theta} d^{\prime}(n e)\right)-\left(1-\left(\theta_{L}+k\left(\bar{\theta}-\theta_{L}\right) d^{\prime}(n e)\right)\left(\bar{\theta}-\theta_{L}\right) d(n e) u^{\prime \prime}\left(\pi_{H}\right)\right.
$$

Since $k>1$ and $1-\bar{\theta} d^{\prime}(n e) \geq 0$, the first term is negative. Also, $\bar{\theta}>\theta_{L}$ implies that $1-$ $\theta_{L} n d^{\prime}(n e)>0$, and, by condition II.2, $\left(1-\left(\theta_{L}+k\left(\bar{\theta}-\theta_{L}\right) d^{\prime}(n e)<0\right.\right.$. Therefore, the second term is negative as well, hence $\frac{\partial \psi}{\partial k}<0$. QED. 
Proof of Proposition 8: Suppose that agents with utility $v$ are more risk averse than those with utility $u$. Then, an increase in risk aversion leads to lower emissions, $e^{N}(v) \leq e^{N}(u)$.

Recall, $\varphi(e, u)=E_{\tilde{\theta}}\left(1-\tilde{\theta} d^{\prime}(n e)\right) u^{\prime}(e-\tilde{\theta} d(n e))$. We will show that $\varphi\left(e^{N}(u), v\right) \leq 0$. Consider a specific value of $\theta$. Either $1-\theta d^{\prime}(n e) \leq 0$, hence $e-\theta d(n e) \leq e-\frac{d(n e)}{d^{\prime}(n e)}$ and $\Phi^{\prime}[u(e-\theta d(n e))] \geq$ $\Phi^{\prime}\left[u\left(e-\frac{d(n e)}{n d^{\prime}(n e)}\right)\right]$ since $u$ is increasing and $\Phi^{\prime}$ is decreasing. This implies that

$$
\left[1-\theta d^{\prime}(n e)\right] \Phi^{\prime}[u(e-\theta d(n e))] \leq\left[1-\theta d^{\prime}(n e)\right] \Phi^{\prime}\left[u\left(e-\frac{d(n e)}{d^{\prime}(n e)}\right)\right]
$$

Or $1-\theta d^{\prime}(n e) \geq 0$, and $\Phi^{\prime}[u(e-\theta d(n e))] \leq \Phi^{\prime}\left[u\left(e-\frac{d(n e)}{n d^{\prime}(n e)}\right)\right]$ which yields the same inequality as above. Since this inequality is valid for any $\theta$, we multiply by $u^{\prime}(e-\theta d(n e))$ and take the expectation. This yields

$$
\varphi(e, v) \leq \Phi^{\prime}\left[u\left(e-\frac{d(n e)}{d^{\prime}(n e)}\right)\right] \varphi(e, u)
$$

At $e=e^{N}(u)$, this reduces to $\varphi\left(e^{N}(u), v\right) \leq 0$. Since $\varphi(e, v)$ starts above the horizontal axis, and crosses it only once at $e^{N}(v)$, we must have $e^{N}(v) \leq e^{N}(u)$. QED.

Proof of Proposition 9: Consider an economy in which agent 1 faces no risk and agent 2 faces a risk. There is a unique equilibrium in which agent 2 does not emit, and agent 1 emits e which solves $1-\bar{\theta} d^{\prime}(e)=0$.

Assume first that agent 1 does not emit, $e_{1}^{N}=0$. Then agent 2 chooses $e_{2}^{N}$ such that

$$
E\left(1-\widetilde{\theta} d^{\prime}\left(e_{2}^{N}\right)\right) u^{\prime}\left(e_{2}^{N}-\widetilde{\theta} d\left(e_{2}^{N}\right)\right)=0
$$

To examine whether this can be an equilibrium let us examine agent 1's best response. Agent 1 then simply chooses $e_{1}$ to maximize $u\left(e_{1}-\bar{\theta} d\left(e_{1}+e_{2}^{N}\right)\right)$ where $e_{2}^{N}$ is characterized by VI.1. So $e_{1}^{N}=0$ would be a best-response if and only if

$$
1-\bar{\theta} d^{\prime}\left(e_{2}^{N}\right) \leq 0
$$

Yet $E\left(1-\tilde{\theta} d^{\prime}\left(e_{2}^{N}\right)\right) u^{\prime}\left(e_{2}^{N}-\tilde{\theta} d\left(e_{2}^{N}\right)\right)$ is equal to

$$
\operatorname{cov}_{\tilde{\theta}}\left[1-\widetilde{\theta} d^{\prime}\left(e_{2}^{N}\right), u^{\prime}\left(e_{2}^{N}-\widetilde{\theta} d\left(e_{2}^{N}\right)\right)\right]+E\left(1-\widetilde{\theta} d^{\prime}\left(e_{2}^{N}\right)\right) E u^{\prime}\left(e_{2}^{N}-\widetilde{\theta} d\left(e_{2}^{N}\right)\right)
$$


which is strictly negative under $u^{\prime}$ strictly decreasing and VI.2. This contradicts VI.1. As a result, there is no equilibrium with $e_{1}^{N}=0$. Let us finally assume that the agent facing uncertainty does not emit at all, $e_{2}^{N}=0$. In that case, agent 1 chooses $e_{1}^{N}$ such that

$$
1-\bar{\theta} d^{\prime}\left(e_{1}^{N}\right)=0
$$

Agent 2 then chooses $e_{2}$ to maximize $W_{N}\left(e_{2}\right) \equiv E u\left(e_{2}-\widetilde{\theta} d\left(e_{1}^{N}+e_{2}\right)\right)$ where $e_{1}^{N}$ is characterized by VI.3. Since $W_{N}$ is concave, a necessary and sufficient condition for $e_{2}^{N}=0$ to be a best response consists in showing that $W_{N}^{\prime}(0) \leq 0$. We get

$$
W_{N}^{\prime}(0)=E\left(1-\widetilde{\theta} d^{\prime}\left(e_{1}^{N}\right)\right) u^{\prime}\left(0-\widetilde{\theta} d\left(e_{1}^{N}\right)\right)=\operatorname{cov}_{\tilde{\theta}}\left[1-\widetilde{\theta} d^{\prime}\left(e_{1}^{N}\right), u^{\prime}\left(0-\widetilde{\theta} d\left(e_{1}^{N}\right)\right]\right.
$$

which is indeed negative under $u^{\prime}$ strictly decreasing. QED.

Proof of Proposition 10: Consider an economy in which agent 1 faces no risk and agent 2 faces a risk. Then, welfare is lower than when both agents face no risk.

Let $e$ be the level of emissions of agent 1 at the Nash equilibrium, which satisfies $1-\bar{\theta} d^{\prime}(e)=0$. Welfare in equilibrium equals $u(e-\bar{\theta} d(e))+E u(0-\tilde{\theta} d(e))$. Under risk aversion, this is lower than $u(e-\bar{\theta} d(e))+u(0-\bar{\theta} d(e))$ which is itself lower than $2 u\left(\frac{1}{2} e-\bar{\theta} d(e)\right)$ by Jensen's inequality. This last quantity is simply the aggregate expected utility that is reached at the symmetric equilibrium under certainty. QED.

Proof of statements in Section IV: We first show that agent 2 emits more than agent 1 in the cooperative case. We denote $\left(e_{1}^{C}, e_{2}^{C}\right)$ the cooperative solution to

$$
\max _{e_{1}, e_{2}} u\left(e_{1}-\bar{\theta} d\left(e_{1}+e_{2}\right)\right)+E_{\tilde{\theta}} u\left(e_{2}-\widetilde{\theta} d\left(e_{1}+e_{2}\right)\right)
$$

After simple manipulations, the condition for an interior cooperative solution $\left(e_{1}^{C}, e_{2}^{C}\right)$ may be written

$$
u^{\prime}\left(e_{1}^{C}-\bar{\theta} d\left(e_{1}^{C}+e_{2}^{C}\right)\right)=E_{\tilde{\theta}} u^{\prime}\left(e_{2}^{C}-\tilde{\theta} d\left(e_{1}^{C}+e_{2}^{C}\right)\right)
$$

which is the standard equalization of expected marginal utilities. Observe that if the marginal utility is convex, $E_{\tilde{\theta}} u^{\prime}\left(e_{2}-\tilde{\theta} d\left(e_{1}+e_{2}\right)\right) \geq u^{\prime}\left(e_{2}-\bar{\theta} d\left(e_{1}+e_{2}\right)\right)$ for any $e_{1}, e_{2}$. Hence under prudence, 
we obtain that agent 2 emits more than agent 1 at the optimum. It can be easily shown that this result holds as well for corner solutions. Namely suppose that one of the two agents emits zero emissions, $e_{i}^{C}=0$. Then, when $u^{\prime \prime \prime}>0$, it must be agent $i=1$.

We now show that, under DARA, the expected utility of agent 2 is higher than that of agent 1. If $u$ is DARA, there exists $T$ increasing and convex such that is $u=T\left(-u^{\prime}\right)$. This implies that $u\left(e_{1}^{C}-\bar{\theta} d\left(e_{1}^{C}+e_{2}^{C}\right)\right)$ which is equal to $T\left(-u^{\prime}\left(e_{1}^{C}-\bar{\theta} d\left(e_{1}^{C}+e_{2}^{C}\right)\right)\right)$ or $T\left(-E u^{\prime}\left(e_{2}^{C}-\tilde{\theta} d\left(e_{1}^{C}+e_{2}^{C}\right)\right)\right)$ is lower than $E\left(T\left(-u^{\prime}\left(e_{2}^{C}-\tilde{\theta} d\left(e_{1}^{C}+e_{2}^{C}\right)\right)\right.\right.$, and so is lower than $E u\left(e_{2}^{C}-\tilde{\theta} d\left(e_{1}^{C}+e_{2}^{C}\right)\right)$.

Proofs of statements in Section V: We first consider the model with uncertain benefits. Denote by $e$ the level of emissions under certainty, solving $\bar{\theta}-d^{\prime}(n e)=0$. We see that: $E(\tilde{\theta}-$ $\left.d^{\prime}(n e)\right) u^{\prime}(\tilde{\theta} e-d(n e))=\operatorname{Cov}\left(\tilde{\theta}-d^{\prime}(n e), u^{\prime}(\tilde{\theta} e-d(n e))\right) \leq 0$, by the definition of covariance and the covariance rule. This implies that emissions under uncertainty are lower than under certainty. Next, introduce $\tilde{\theta}=\bar{\theta}+k \tilde{\varepsilon}$. Following the same methodology as in the proof of Proposition 5, we can show that $e^{\prime}(0)=W^{\prime}(0)=0$ and $W^{\prime \prime}(0)=\frac{2(n-1)}{n}\left(-u^{\prime \prime}\right) V e(0)\left[\frac{d^{\prime}}{d^{\prime \prime}}-\frac{n}{2(n-1)}\right]$. Uncertainty improves welfare for small risks if $d^{\prime} / d^{\prime \prime}>n /(2(n-1))$. This is satisfied, for instance, if $d(e)=C(\exp (D e)-1)$ and $D<2(n-1) / n$.

We now consider the model with two periods. Without loss of generality, suppose that $w_{0}=w_{1}=0$, and denote again by $e$ the level of emissions under certainty. It now solves $u_{0}^{\prime}(e)-d^{\prime}(n e) \bar{\theta} u_{1}^{\prime}(-\bar{\theta} d(n e))=0$. We have: $u_{0}^{\prime}(e)-d^{\prime}(n e) E \tilde{\theta} u_{1}^{\prime}(-\tilde{\theta} d(n e))=d^{\prime}(n e)\left[\bar{\theta} u_{1}^{\prime}(-\bar{\theta} d(n e))-\right.$ $\left.E \tilde{\theta} u_{1}^{\prime}(-\tilde{\theta} d(n e))\right]=d^{\prime}(n e)\left[\bar{\theta} u_{1}^{\prime}(-\bar{\theta} d(n e))-\bar{\theta} E u_{1}^{\prime}(-\tilde{\theta} d(n e))-\operatorname{Cov}\left(\tilde{\theta}, u_{1}^{\prime}(-\tilde{\theta} d(n e))\right)\right]$. Then,

$\operatorname{Cov}\left(\tilde{\theta}, u_{1}^{\prime}(-\tilde{\theta} d(n e))\right) \geq 0$ by the covariance rule. In addition, $E u_{1}^{\prime}(-\tilde{\theta} d(n e)) \geq u_{1}^{\prime}(-\bar{\theta} d(n e))$ if $u_{1}^{\prime}$ convex. Thus, $u_{0}^{\prime}(e)-d^{\prime}(n e) E \tilde{\theta} u_{1}^{\prime}(-\tilde{\theta} d(n e)) \leq 0$ under prudence, which implies that emissions under uncertainty are lower than under certainty. The analysis of small risks yields, again, that $e^{\prime}(0)=W^{\prime}(0)=0$. The expression for $W^{\prime \prime}(0)$ is more complicated. Omitting arguments for clarity, we find that $W^{\prime \prime}(0)=(n-1) V \bar{\theta} d d^{\prime 2} \frac{-2 u_{1}^{\prime \prime}+\bar{\theta} d u_{1}^{\prime \prime \prime}}{n \bar{\theta} d^{\prime \prime} u_{1}^{\prime}-n \bar{\theta}^{2} d^{\prime 2} u_{1}^{\prime \prime}-u_{0}^{\prime \prime}}+V d^{2} u_{1}^{\prime \prime}$. The first term corresponds to the indirect effect of changed emissions, and is positive under prudence. The second term captures the direct, negative effect of uncertainty. For example when $u$ satisfies CARA and $d(e)=D e$, we can see that a symmetric equilibrium exists, and uncertainty improves welfare if $D$ small enough. 


\section{References}

Arrow, Kenneth J., 1963, Uncertainty and the welfare economics of medical care, American Economic Review, 53, 941-73.

Arrow, Kenneth J. and Robert C. Lind, 1970, Uncertainty and the evaluation of public investment decisions, American Economic Review, 60, 364-78.

Azar, Christian and Henning Rodhe, 1997, Targets for stabilization of atmospheric CO2, Science, $276,1818-19$.

Barrett, Scott, 2007. Why Cooperate? The Incentive to Supply Global Public Goods. Oxford: Oxford University Press.

Baker, Erin, 2005, Uncertainty and learning in a strategic environment: Global climate change, Resource and Energy Economics, 27, 19-40.

Boucher, Vincent and Yann Bramoullé, 2007, Risk aversion and international environmental agreements, mimeo, Laval University, Québec, Canada.

Bramoullé, Yann and Nicolas Treich, 2004, Can uncertainty alleviate the commons problem?, mimeo, LERNA, Toulouse School of Economics, France.

Eso, Peter and Lucy White, 2004, Precautionary bidding in auctions, Econometrica, 72, 77-92.

Gollier, Christian, 2001, The Economics of Risk and Time, The MIT Press.

Gradstein, Mark, Nitzan, Shmuel and Steven Slutsky, 1992, The effect of uncertainty on interactive behavior, Economic Journal, 102, 554-61.

Hadar, Josef and Tae Kun Seo, 1990, The effects of shifts in a return distribution on optimal portfolios, International Economic Review, 31, 721-36.

Hammitt, James K. and John L. Adams, 1996, The value of international cooperation for abating global climate change, Resource and Energy Economics, 18, 219-41.

Heal, Geoffrey and Bengt Kriström, 2002, Uncertainty and climate change, Environmental and Resource Economics, 22, 3-39.

Intergovernmental Panel on Climate Change (IPCC), 1995, Working Group 3, Economic and Social Dimensions of Climate Change, Cambridge University Press.

Intergovernmental Panel on Climate Change (IPCC), 2007, Climate Change 200\%: The Physical Basis. Contribution of Working Group I to the Fourth Assessment Report of IPCC, Cambridge University Press, Cambridge, United Kingdom and New York, NY, USA. 
Kimball, A. W., 1951, On dependent tests of significance in the analysis of variance, The Annals of Mathematical Statistics, 22, 600-602.

Kimball, Miles, 1990, Precautionary savings in the small and in the large, Econometrica, 58, 53-73.

Kolstad, Charles, 2005, Piercing the veil of uncertainty in transboundary pollution agreements, Environmental and Resource Economics, 31, 21-34.

Na, Seong-lin and Hyun Song Shin, 1998, International environmental agreements under uncertainty, Oxford Economic Papers, 50, 141-70.

Nordhaus, William D., 1994, Managing the Global Commons, The Economics of Climate Change, MIT Press.

Peltzman, Sam, 1975, The effects of automobile safety regulation, Journal of Political Economy, $83,677-725$.

Pratt, John W., 1964, Risk aversion in the small and in the large, Econometrica, 32, 122-36.

Rothschild, Michael and Joseph Stiglitz, 1970, Increasing risk: I. A definition, Journal of Economic Theory, 2, 225-43.

Sandler, Todd, Sterbenz Frederic P. and John Posnett, 1987, Free riding and uncertainty, European Economic Review, 31, 1605-17.

Sandler, Todd and Frederic P. Sterbenz, 1990, Harvest uncertainty and the Tragedy of the Commons, Journal of Environmental Economics and Management, 18, 155-67.

Stern, Nicholas et al., 2007, The Economics of Climate Change, Cambridge University Press.

Ulph, Alistair and David Maddison, 1997, Uncertainty, learning and international environmental policy coordination, Environmental and Resource Economics, 9, 451-66.

Ulph, Alistair and David Ulph, 1996, Who gains from learning about global warming?, in Economics of Atmospheric Pollution, E. van Ierland and K. Gorka eds, NATO ASI series.

Ulph, Alistair, 2004, Stable environmental international agreements with a stock pollutant, uncertainty and learning, Journal of Risk and Uncertainty, 29, 53-73.

Viscusi, W. Kip, 1984, The lulling effect: The impact of child-resistant packaging on aspirin and analgesic ingestion, American Economic Review, Papers and Proceedings, 74, 324-27.

Viscusi, W. Kip, 1994, Mortality effects of regulatory costs and policy evaluation criteria, Rand Journal of Economics, 25, 94-109. 
Weitzman, Martin L., 2007, A review of the Stern review on the Economics of Climate Change, Journal of Economic Literature, 45, 703-724.

White, Lucy, 2004, Prudence in bargaining: The effect of uncertainty on bargaining outcomes, Games and Economic Behavior, forthcoming.

Young, Oran, 1994, International Governance: Protecting the Environment in a Stateles Society, Cornell University Press, Ithaca. 


\section{Table 1. Effect of greenhouse gas concentration on global mean temperature increase.}

Reproduces the findings of different studies on the equilibrium temperature projections for different levels of greenhouse gas concentration using 5\%-95\% climate sensitivity ranges, see Table 1.1. in Stern et al. (2007, p. 12) for details and references.

\begin{tabular}{|c|c|c|c|}
\hline \multirow{2}{*}{$\begin{array}{l}\text { Stabilisation level } \\
\text { ppm CO2 equivalent) }\end{array}$} & \multicolumn{3}{|l|}{ Temperature increase at equilibrium relative to pre-industrial $\left({ }^{\circ} \mathrm{C}\right)$} \\
\cline { 2 - 4 } & IPCC TAR 2001 & Hadley Centre & Eleven Studies \\
\hline 400 & $0.8-2.4$ & $1.3-2.8$ & $0.6-4.9$ \\
\hline 450 & $1.0-3.1$ & $1.7-3.7$ & $0.8-6.4$ \\
\hline 500 & $1.3-3.8$ & $2.0-4.5$ & $1.0-7.9$ \\
\hline 550 & $1.5-4.4$ & $2.4-5.3$ & $1.2-9.1$ \\
\hline 650 & $1.8-5.5$ & $2.9-6.6$ & $1.5-11.4$ \\
\hline 750 & $2.2-6.4$ & $3.4-7.7$ & $1.7-13.3$ \\
\hline 1000 & $2.8-8.3$ & $4.4-9.9$ & $2.2-17.1$ \\
\hline
\end{tabular}


Figure 1. Increase in catastrophic risk and emissions

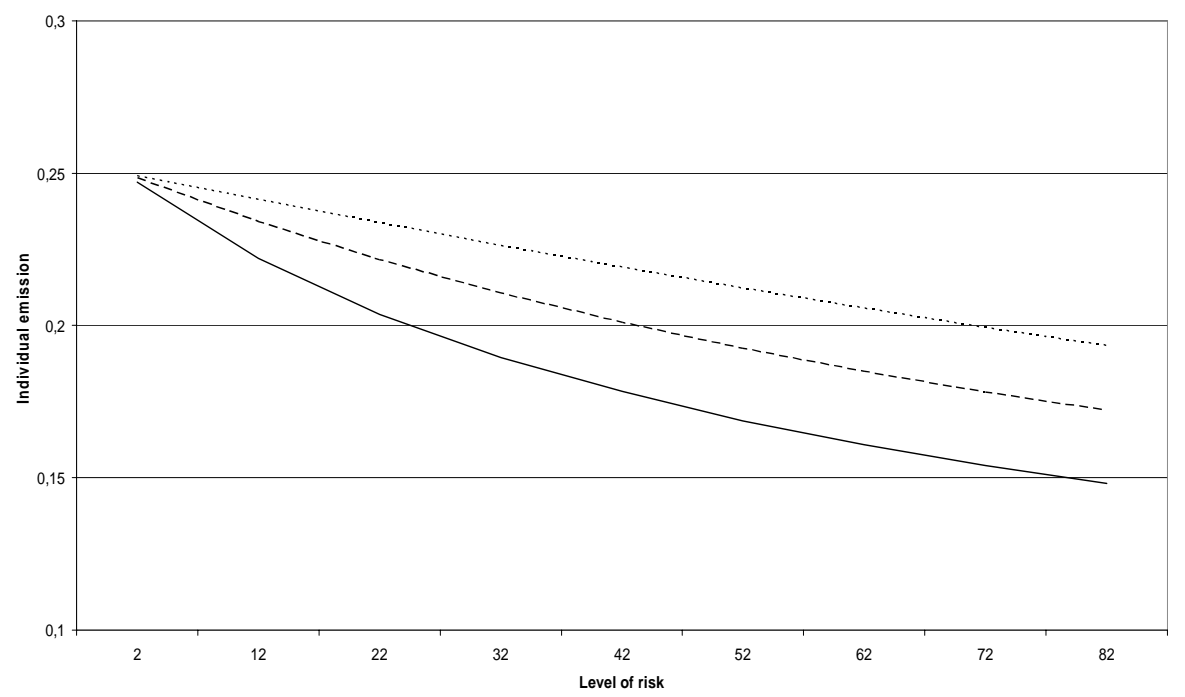

Figure 2. Increase in catastrophic risk and welfare

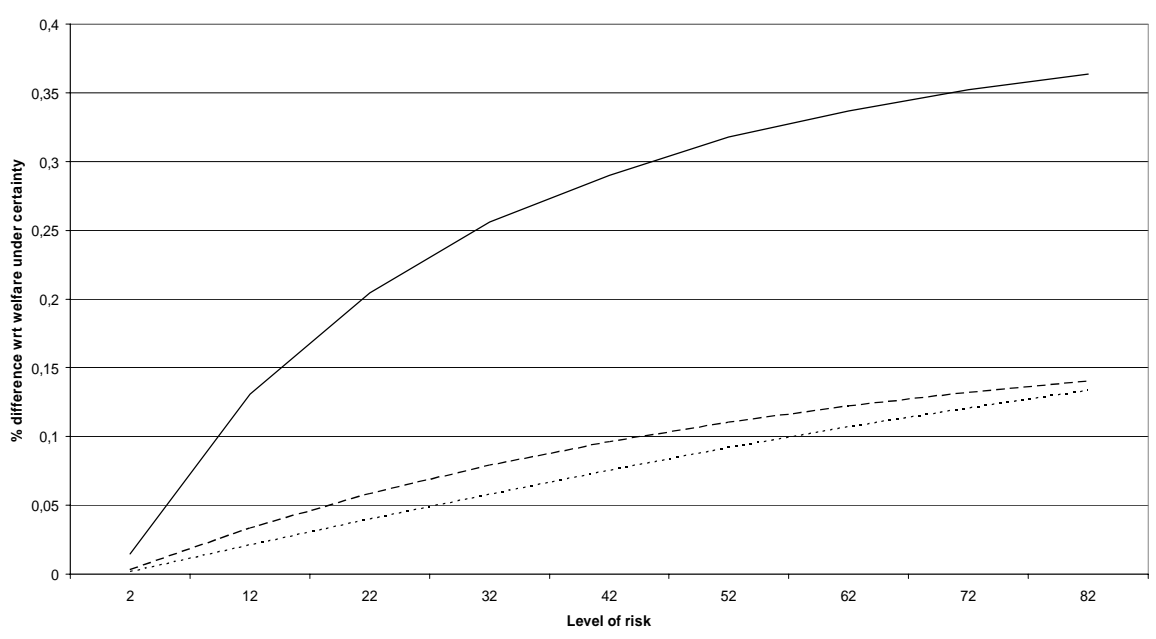

$\begin{array}{ll}\gamma & =1 / 2 \\ \cdots & \gamma=1 \\ \cdots & \gamma=2\end{array}$

